\title{
PROGRESS AND ISSUES IN THE ELECTROMAGNETIC PRODUCTION OF KAON ON THE NUCLEON
}

\author{
T. MART \\ Departemen Fisika, FMIPA, Universitas Indonesia Depok 16426, Indonesia \\ tmart@fisika.ui.ac.id \\ The present status of kaon photo- and electroproduction on the nucleon is \\ briefly reviewed. Some current important issues in this field are discussed. \\ Keywords: Kaon, photoproduction, electroproduction, nucleon resonance
}

\section{Introduction}

Compared to the case of pions the electromagnetic productions of kaons are less understood. This is due to the fact that the strangeness quantum number is explicitly present in the final state of the process. Although the reactions involving kaons are slightly more complicated, the additional degrees of freedom created by the strangeness can give information not available from nucleons and pions. For instance, due to the conservation of the strangeness, the production of kaons is always accompanied by the creation of hyperons, which can be used to deeply explore the structure of the nucleus since they are not blocked by the Pauli principle. On the other hand, this elementary process provides an important input for the calculation of hypernuleus photo- or electroproduction. Along with the fact that some $N^{*}$ resonances that were predicted in quark models have only noticeable branching ratios into the $K \Lambda$ channel, kaon photo- and electroproduction clearly have drawn many attentions for more than five decades.

\section{Theoretical Models}

The earliest attempt to theoretically explain kaon photo- and electroproduction was proposed by Kawaguchi and Moravcsik more than 50 years ago ${ }^{1}$ Interestingly, all of the six possible isospin channels were already considered by utilizing only three Feynman diagrams of the Born terms, 
though hitherto no experimental data were available. The results were obviously very modest and, in fact, the cross sections for the $K^{0} \Lambda$ and $K^{0} \Sigma^{0}$ channels were predicted to be zero since neither nucleon anomalous magnetic moments nor resonance contributions were taken into account. Since that time considerable efforts were devoted to explain the appearing experimental data, although only Thom who tried to seriously fit the data to an isobar model. After that, there were a number of works using isobar model,$^{2}$ dispersion relation,,$^{3,4}$ multipoles analysis,${ }^{5}$ and Regge approach .6 After the mid-seventies, the interest in this field was temporarily dormant, mainly due to the lack of experimental facilities.

The interest in kaon photo- and electroproduction was revived by the constructions of modern accelerators such as those in JLAB, MAMI, ELSA, and others laboratories. The work of Adelseck, Bennhold, and Wright started the new era of phenomenological models in $K^{+} \Lambda$ photoproduction . ${ }^{7}$ This work was refined with the inclusion of more data and electroproduction process ${ }^{8}$ and extended to all isospin channels. ${ }^{9}$ A chiral quark model with less parameters has been also put forward ${ }^{10}$ in an attempt to recover the low energy theorem. The results of this model are modest and, in fact, the $K^{0} \Sigma^{+}$cross section is predicted to be larger than that for $K^{+} \Sigma^{0}$ by a factor of approximately two, since contributions from the seagull and $s$-channel resonance have opposite signs.

Modern calculations of kaon photoproduction exploit chiral perturbation theory ${ }^{11}$ and coupled channels analysis.$^{12,13}$ In the higher energy regions, the Regge ${ }^{14}$ or hybrid models ${ }^{15}$ (a combination of the Feynman diagrammatic technique and Regge formalism) turn out to be more appropriate. Nevertheless, for practical use such as for nuclear applications and other phenomenological studies, the single channel isobaric analysis is still proven to be powerful..$^{9,16,17}$

\section{Experimental Data}

Historically, experimental data of kaon photo- and electroproduction can be divided into two categories, old data (published before 1980) and new data (published after 1990). A list of references for old data is given, e.g., in Refs. ${ }^{18,19}$ whereas the corresponding kinematical coverage for the photoproduction is shown in the left panel of Fig. 1. Surprisingly, the old data cover also forward angle regions, the case which is very difficult to achieve with the presently available technologies, as obviously shown in the right panel of Fig. 1. As discussed in the following section, this region is in fact very decisive for constraining the models and for nuclear applications. Nev- 

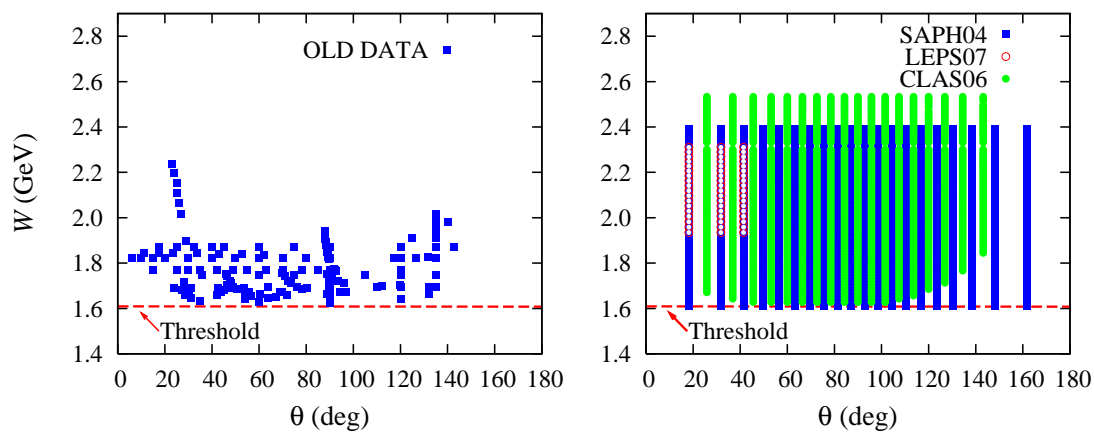

Fig. 1. Kinematical coverages of the experimental data from old databases (left) and new experiments (right) for the $\gamma p \rightarrow K^{+} \Lambda$ differential cross section. In the right panel data from SAPHIR ${ }^{20}$ (SAPH04), CLAS ${ }^{21}$ (CLAS06), and LEPS ${ }^{22}$ (LEPS07) collaborations are shown.

ertheless, the data quality is in general poor and, as a consequence, the cross sections data do not show resonance structures. There were few data available for the recoiled $\Lambda$ polarization and two data points on the target asymmetry.

Kaon photoproduction started to become more interesting after the publication of SAPHIR data ${ }^{19}$ on the $K^{+} \Lambda$ and $K^{+} \Sigma^{0}$ channels in 1998, since the corresponding error bars allow for an identification of resonance structures, especially in the $K^{+} \Lambda$ channels. Further analysis of SAPHIR data ${ }^{20}$ as well as the new CLAS data ${ }^{21}$ confirmed these structures. Note that in both SAPHIR and CLAS data sets, there are plenty of data on the recoil polarization. Together with the new data from the LEPS ${ }^{22,23}$ and GRAAL ${ }^{24}$ collaborations, these data provide a strong constraint on the proliferation of phenomenological models. In the $K \Sigma$ channels, new data have also appeared from SAPHIR collaboration ${ }^{25}\left(K^{0} \Sigma^{+}\right)$, and from the LEPS collaboration ${ }^{26}\left(K^{+} \Sigma^{-}\right)$. The ability to reverse the direction of photon helicity in the SPRING8 has led to the measurements of the photon asymmetry $\Sigma$ in the $K^{+} \Lambda, K^{+} \Sigma^{0}$, as well as $K^{+} \Sigma^{-}$channels ${ }^{22,26}$

Since the polarization of the recoiled $\Lambda$ can be obtained without any additional apparatus, measurements of the double polarization observables $C_{x}$ and $C_{z}$ (or $O_{x}$ and $O_{z}$ ) are possible, provided that the polarized photon beams are available. The first measurement of these observables was performed by the CLAS collaboration ${ }^{27}$ and because the available beams at JLAB are circularly polarized, the corresponding observables are $C_{x}$ and $C_{z}$. Very recently, by utilizing the linearly polarized photon the GRAAL 


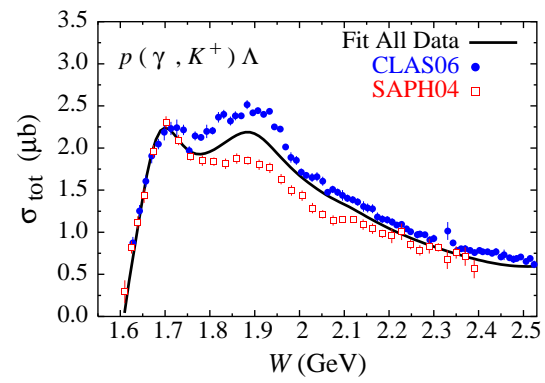

Fig. 2. The apparent difference between the CLAS ${ }^{21}$ and SAPHIR ${ }^{20}$ data compared with the result of fitting a multipoles model ${ }^{32}$ to both data sets.

collaboration has been able to measure the $O_{x}$ and $O_{z}$ observables ${ }^{28}$ from threshold up to $E_{\gamma}=1.5 \mathrm{GeV}$.

The electroproduction experiments with high statistics have been also performed at JLAB.${ }^{29-31}$ It is reported that the longitudinal and transverse components of the cross section can be nicely separated, ${ }^{29,31}$ whereas the transferred polarization in the $\overrightarrow{e p} \rightarrow e^{\prime} K^{+} \vec{\Lambda}$ process has been measured.$^{30}$

\section{Current Important Issues in Kaon Photoproduction}

\subsection{The problem of data discrepancy}

In spite of their unprecedented high statistics, the new CLAS ${ }^{21}$ and SAPHIR $^{20}$ data reveal a lack of mutual consistency in the forward and backward regions. This problem can be summarized by the total cross section shown in Fig. 2, where it is obvious that fitting a phenomenological model to both data sets simultaneously results in a model which is inconsistent to all data sets. As shown by Ref. ${ }^{32}$ this problem also hinders a reliable extraction of the resonance parameters, since the use of SAPHIR and CLAS data, individually or simultaneously, leads to quite different resonance parameters. Therefore, the presently available data do not allow for a firm conclusion on the extracted "missing resonances".

By using four different isobar models Ref. ${ }^{33}$ studied the statistical properties of both CLAS and SAPHIR data. It is found that the SAPHIR data are coherently shifted down with respect to the CLAS and LEPS ${ }^{22}$ data, especially at forward kaon angles. A global scaling factor of $15 \%$ is required to remove this discrepancy. The phenomenon also implies that the LEPS data are more consistent with the CLAS data than the SAPHIR ones. Interestingly, at forward angles both CLAS and LEPS data sets can be 

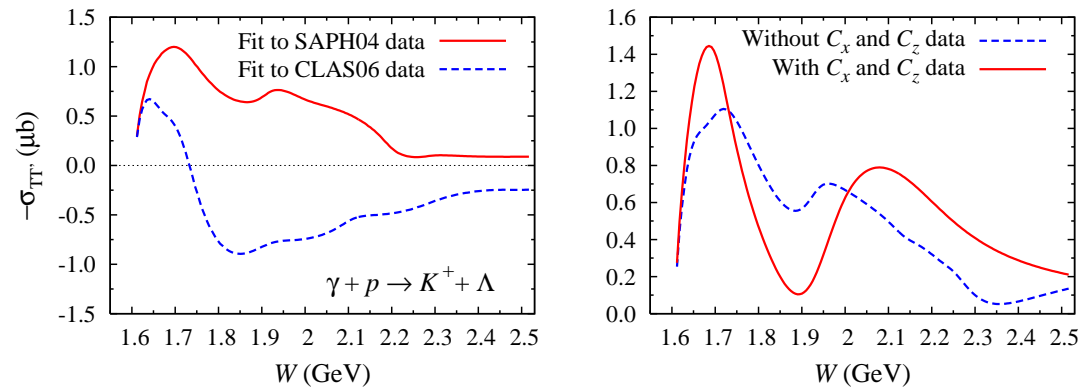

Fig. 3. Variations of the total cross section $\sigma_{\mathrm{TT}^{\prime}}$ obtained from fitting to different experimental data sets (left) and fitting to the data sets that include and exclude the beam-recoil polarization $C_{x}$ and $C_{z}$ data (see Ref. ${ }^{34}$ for details).

described reasonably well within the isobaric model without hadronic form factors. This can be understood from the energy distribution of the cross sections at this kinematics, as will be discussed in the following subsections. The statistical study recommend that a combination of CLAS, LEPS, and old $^{\mathrm{a}}$ data sets in the fitting data base is the more preferred choice, while a combination of CLAS and SAPHIR data should not be assumed in fixing parameters of models, especially at forward angles.

Despite considerable efforts to alleviate this problem, it is still difficult to determine which data set should be used to obtain a reliable phenomenological model. The reason is that in all analyses the experimental data are fitted by adjusting a set of free parameters, while the precise values of these parameters are not well known. Furthermore, the extracted parameters are not unique and also sensitive to the number of resonances used in a model. In view of this, Ref. ${ }^{34}$ proposed to use other quantities which can be predicted by the models and can be directly compared with the results from other measurements or model predictions. One of the possible quantities is the contribution of the $\gamma p \rightarrow K^{+} \Lambda$ channel to the GerasimovDrell-Hearn (GDH) sum rule, ${ }^{35}$ which relates the anomalous magnetic moment of the proton $\kappa_{p}$ to the total photoabsorption cross sections $\sigma_{\mathrm{TT}^{\prime}}$, i.e. $\kappa_{p}^{2}=\left(m_{p}^{2} / \pi^{2} \alpha\right) \int_{0}^{\infty} d E_{\gamma} \sigma_{\mathrm{TT}^{\prime}} / E_{\gamma}$. By looking at the energy distributions of the $\sigma_{\mathrm{TT}^{\prime}}$ obtained from models that fit to CLAS and SAPHIR data shown in the left panel of Fig. 3, it is obvious that the two data sets lead to different contribution to $\kappa_{p}$. It is then concluded that contribution from the model that fits to the SAPHIR data sets is more preferred ${ }^{34}$ Nevertheless,

${ }^{a}$ see Section 3 for the definition of old data 
experimental measurement of the $\sigma_{\mathrm{TT}^{\prime}}$ is clearly required to confirm this claim. Since the SAPHIR detector has been dismantled, measurements of the $\sigma_{\mathrm{TT}^{\prime}}$ by the CLAS or MAMI collaboration seem to be the only choice.

\subsection{The effects of $C_{x}$ and $C_{z}$ data}

The beam-recoil polarization observables data, $C_{x}$ and $C_{z}$, published by the CLAS collaboration ${ }^{27}$ recently, indicate that the $\Lambda$ polarization is predominantly in the direction of the spin of the incoming photon. It is interesting that recent analyses found that these data seems to be difficult to explain. In Ref. ${ }^{34}$ it is reported that the inclusion of these data reduces the complicated structure of the total cross sections $\sigma_{\mathrm{TT}^{\prime}}$ as shown in the right panel of Fig. 3, which indicates that the $C_{x}$ and $C_{z}$ data select only certain important resonances, i.e. the $S_{11}(1650), P_{11}(1710), P_{13}(1720)$, and $P_{13}(1900)$ resonances. This finding corroborates the result of a recent coupled channels analysis. ${ }^{36}$

\subsection{The second peak in the cross section}

The first version of SAPHIR data on $\gamma p \rightarrow K^{+} \Lambda$ released in 1994 did not give any hint about resonance structures in the cross section, since the statistics was very limited ${ }^{37}$ However, by collecting more statistics the second version of SAPHIR data ${ }^{19}$ released in 1998 display two distinct peaks in the total cross section at $W \approx 1700 \mathrm{MeV}$ and $1900 \mathrm{MeV}$. More accurate data published by the SAPHIR ${ }^{20}$ and CLAS $^{21}$ collaborations 5 and 8 years later, respectively, confirm the existence of the two peaks (see Fig. 2). By comparing the extracted resonance widths and branching fractions with the predictions of a constituent quark model ${ }^{38}$ Ref. ${ }^{16}$ concluded that the second peak corresponds to the $D_{13}(1895)$ nucleon resonance that is missing from the PDG list. ${ }^{39}$ Although many subsequent analyses used this spin $3 / 2$ nucleon resonance in their models, there was a suggestion ${ }^{40}$ that this peak could come from other resonances such as $S_{11}, P_{11}$ or $P_{13}$. However, the possibility of using these resonances, instead of the $D_{13}(1895)$, has been also discussed in Ref. ${ }^{16}$ but it was ruled out after comparing the extracted widths and branching fractions with the predictions of quark model. Further study using a multipoles approach ${ }^{32}$ found that, in spite of their differences, both SAPHIR ${ }^{20}$ and CLAS ${ }^{21}$ data sets indicate that the peak originates from the $D_{13}$ resonance with a mass between $1911-1936$ $\mathrm{MeV}$. The necessity of the $D_{13}$ in the analyses of $K^{+} \Lambda$ photoproduction with a mass of around $1900 \mathrm{MeV}$ has been also corroborated by recent cou- 


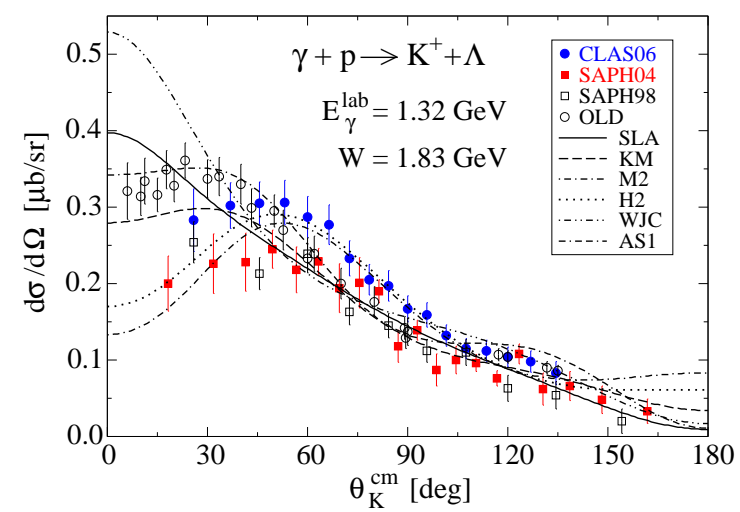

Fig. 4. Variations of different models and experimental data at forward angles ${ }^{33}$

pled channels analyses ${ }^{13}$ Note that in these analyses the recoil polarization data have been also taken into account. It is quite interesting, however, that a recent effective field theory analysis suggests that the peak should correspond to the $P_{11}$ state which is a bound state of $K \bar{K} N$ with a mixture of $a_{0}(980) N$ and $f_{0}(980) N$ components ${ }^{41}$ To confirm this idea, two experiments are proposed, i.e. first to exclude contributions of the spin $3 / 2$ states and second to find an indication of the $K \bar{K} N$ bound state. On the other hand, a preliminary study ${ }^{42}$ performed by constraining the free parameters in a multipole analysis ${ }^{32}$ to the PDG values indicates that the peak originates from the contributions of both $S_{11}$ and $P_{11}$ states with masses around $1920 \mathrm{MeV}$. In this study it is found that the contribution of the $S_{11}$ state is stronger than that of the $P_{11}$ and the result does not depend on which data set is being used in the fitting database.

\subsection{The problem at forward angles}

Since the dominant contributions to the total cross section shown in Fig. 2 come from the forward angles data, the largest discrepancies between the CLAS and SAPHIR data obviously show up at the forward angles. Unfortunately, the available phenomenological models vary wildly at these kinematical regions. This situation is clearly shown in Fig. 4, in which we can see that for $0^{\circ} \leq \theta_{K} \leq 30^{\circ}$ our best knowledge on kaon photoproduction cannot tell us about the actual values of differential cross sections.

Meanwhile, theoretical predictions of the hypernuclear photo- or electroproduction rely heavily on the elementary operator extracted from kaon 

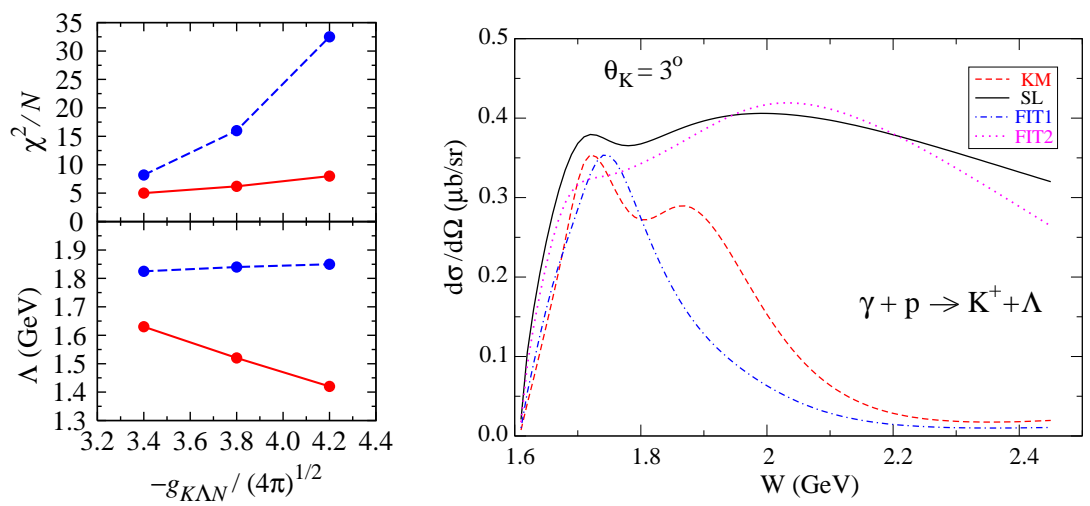

Fig. 5. (Left panel) Variations of the $\chi^{2} / N$ and the cutoff parameter of hadronic form factor $\Lambda$ due to the variation of the leading coupling constant $g_{K \Lambda N} .{ }^{46}$ Solid lines show results obtained with the Haberzettl's method ${ }^{45}$ whereas dashed lines display the Ohta's prescription. ${ }^{44}$ (Right panel) Effects of the hadronic form factor on the energy distribution of the differential cross section ${ }^{33}$ for different phenomenological models. Models of KM and FIT1 utilize the hadronic form factor, while SL and FIT2 exclude this form factor (see Ref. ${ }^{33}$ for further explanation of the used models).

photo- or electroproduction data at very forward angles. As an example, in the electromagnetic production of the hypertriton, the magnitude of differential cross section is only realistic for experimental measurement at $0^{\circ} \leq \theta_{K} \leq 20^{\circ}$, whereas at $\theta_{K} \approx 25^{\circ}$ the cross section is practically zero..$^{43}$ In view of this, accurate and reliable experimental data as well as theoretical formulation of kaon photoproduction in the forward region are the first condition toward a reliable prediction of the hypernuclear photoproduction cross section.

\subsection{The controversy of hadronic form factors}

It has been well known that the background terms become divergent at high energies. Therefore, the use of hadronic form factors to reduce the background contribution is desired. It is also known that the use of this form factor leads to the violation of gauge invariance of the amplitude. As a result, several methods have been put forward in the last decades to overcome this problem. Two of the well known prescriptions are due to Ohta ${ }^{44}$ and Haberzettl. ${ }^{45}$ As can be seen from the left panel of Fig. 5, the latter is superior since it can provide a reasonable description of experimental data, i.e. producing relatively much smaller $\chi^{2}$, using values for the leading couplings constants close to the $\mathrm{SU}(3)$ values. In Ohta's method this is not 


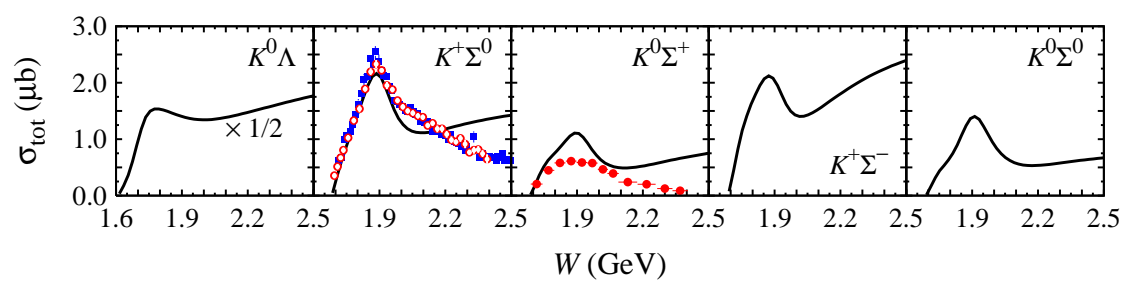

Fig. 6. Total cross sections for the rest 5 isospin channels of kaon photoproduction on the nucleon. Solid lines show the calculated cross sections of Kaon-Maid , ${ }^{47}$ whereas solid squares, open circles, and solid circles display experimental data from Refs. $,{ }^{21},{ }^{20}$ and, ${ }^{25}$ respectively. Note that experimental data in the $K^{+} \Sigma^{-}$channel are only available in the differential cross section ${ }^{26}$ whereas the cross section of the $K^{0} \Lambda$ channel has been renormalized by a factor of $1 / 2$ in order to fit on the scale.

possible due to the absence of a hadronic form factor in the electric current contribution.

Since then, most of the phenomenological models include hadronic form factors in their background terms. On the other hand, Ref. ${ }^{40}$ proposed to use certain hyperon resonances, the $S_{01}(1800)$ and $P_{01}(1810)$, for counterbalancing the strength of the Born terms. This is achieved through a destructive interference between these $u$-channel resonances and the Born terms, thereby reducing the strength to a realistic level. However, the method is not so convincing for the huge number of presently available experimental data and, on the other hand, the use of hadronic form factor is more flexible and simultaneously takes into account the fact that nucleons are composite objects, and not point-like.

Recently, Ref. ${ }^{33}$ has demonstrated that the behavior of forward-angle cross sections as a function of the energy for models that include the hadronic form factors is far from realistic. This finding is depicted in the right panel of Fig. 5, where the hadronic form factors drastically suppress the cross sections at $W \geq 2 \mathrm{GeV}$. Although no data are available for comparison at this kinematics, such a strong damping has not been observed in the experimental data near the forward angles. As can be seen in Figs. 4 and 6 of Ref. ${ }^{32}$ differential cross sections for $\theta_{K}=18^{\circ}-37^{\circ}$ tend to be flat up to $W=2.5 \mathrm{GeV}$. Therefore, the concept of hadronic form factors in meson photoproduction needs to be revisited in the future.

\subsection{Other isospin channels}

By considering the conservation of quantum numbers, there are six possible reaction channels for kaon photoproduction on the nucleon, three on the 


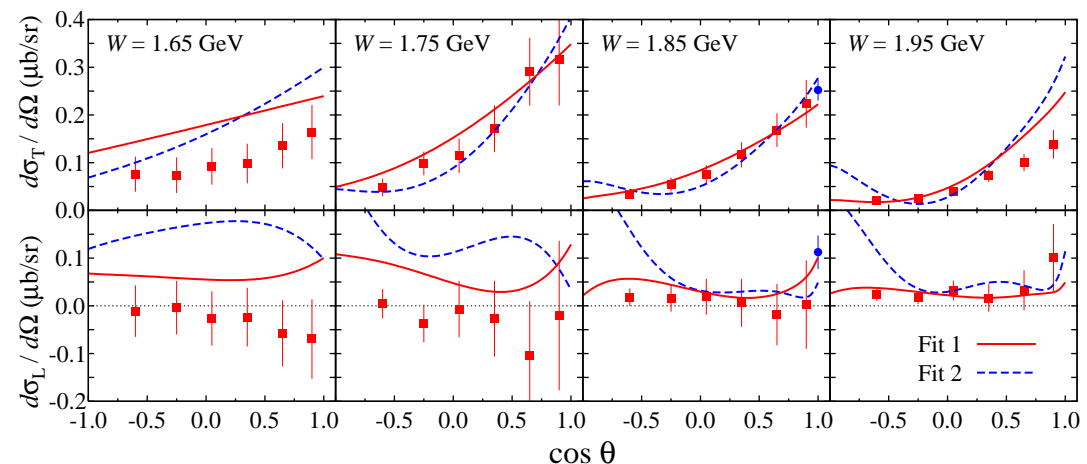

Fig. 7. Longitudinal and transverse differential cross sections of the $e p \rightarrow e^{\prime} K^{+} \Lambda$ process. The CLAS data (solid squares ${ }^{31}$ and solid circles ${ }^{29}$ ) are compared with the results from two multipoles models Fit 1 and Fit 2 (see Ref. ${ }^{51}$ for details).

proton and three on the neutron. Except for the $\gamma p \rightarrow K^{+} \Lambda$ channel, these possible reactions are shown in Fig. 6, where the calculations obtained from Kaon-Maid are compared with the available data. Note that there are few data points available just recently for the $\gamma n \rightarrow K^{+} \Sigma^{-}$differential cross section released by the LEPS collaboration. ${ }^{26}$ Thus, there are no data for the neutral kaon photoproduction on the neutron, $\gamma n \rightarrow K^{0} \Lambda$ and $\gamma n \rightarrow K^{0} \Sigma^{0}$, although from Fig. 6 it is clear that the data can severely constrain the model, especially in the $\gamma n \rightarrow K^{0} \Lambda$ channel. Furthermore, the investigation of the $Y N$ potentials through the $\gamma d \rightarrow K Y N$ reaction requires the information from these two channels ${ }^{48}$ We note that important progress in this direction has been indicated by the newly published $\gamma d \rightarrow$ $K^{0} K Y$ data by the Tohoku's group ${ }^{49}$ It is also important to note that the $K^{0}$ photoproduction excludes the $t$-channel in the Born terms. Thus, by comparing photoproductions of $K^{+}$and $K^{0}$, one can study the influence of the kaon propagator in kaon photoproduction.

\subsection{Kaon electroproduction}

It is widely known that the electroproduction process can give more information not available from the photoproduction one. One of the important information is the electromagnetic structure (form factor) of the kaon, which is hiding in the longitudinal cross section $\left(d \sigma_{\mathrm{L}} / d \Omega\right)$. In contrast to the pion case, where the mass of pion is much smaller and therefore the $t$-channel can dominate the process, so that an independent extraction is possible,${ }^{50}$ the extraction of kaon electromagnetic form factor requires a 
reliable phenomenological model. Consequently, accurate measurements of $d \sigma_{\mathrm{L}} / d \Omega$ are required for this purpose. However, as seen from the lower panels of Fig. 7, the presently available data still do not allow for the extraction of this form factor. Furthermore, to suppress contaminations from nucleon resonances, measurements with $W \geq 2.4 \mathrm{GeV}$ are recommended.

\section{Summary and Conclusion}

The recent progress and some important issues in photo- and electroproduction of kaon on the nucleon have been briefly presented. It is quite apparent that more experimental and theoretical works are needed to settle the present problems in this field. Nevertheless, with the modern concept of accelerator and spectrometer technologies available at JLAB, MAMI, ELSA, SPRING8, and other laboratories, we are quite optimistic that these problems can be solved in the near future.

\section{Acknowledgments}

The author acknowledges the support from the Universitas Indonesia.

\section{References}

1. M. Kawaguchi and M. J. Moravcsik, Phys. Rev. 107, 563 (1957).

2. B. B. Deo and A. K. Bisoi, Phys. Rev. D 9, 288 (1974).

3. N. F. Nelipa, Nucl. Phys. 82, 680 (1966).

4. A. R. Pickering, Nucl. Phys. B66, 493 (1973).

5. W. Schorsch, J. Tietge, and W. Weilnböck, Nucl. Phys. B25, 179 (1970).

6. N. Levy, W. Majerotto, and B. J. Read, Nucl. Phys. B55, 493 (1973);B55, 513 (1973).

7. R. A. Adelseck, C. Bennhold, and L. E. Wright, Phys. Rev. C 32, 1681 (1985).

8. R. A. Adelseck and B. Saghai, Phys. Rev. C 42, 108 (1990); J.-C. David, C. Fayard, G. H. Lamot, and B. Saghai, Phys. Rev. C 53, 2613 (1996).

9. T. Mart, C. Bennhold, and C. E. Hyde-Wright, Phys. Rev. C 51, R1074 (1995); T. Mart and C. Bennhold, Nucl. Phys. A 585, 369c (1995).

10. Zhenping Li, Phys. Rev. C 52, 1648 (1995).

11. S. Steininger and U. G. Meissner, Phys. Lett. B 391, 446 (1997); B. Borasoy, P. C. Bruns, U. G. Meissner and R. Nissler, Eur. Phys. J. A 34, 161 (2007).

12. N. Kaiser, T. Waas and W. Weise, Nucl. Phys. A 612, 297 (1997); T. Feuster and U. Mosel, Phys. Rev. C 59, 460 (1999).

13. A. V. Anisovich et al., Eur. Phys. J. A 25, 427 (2005); B. Julia-Diaz, B. Saghai, T. S. Lee and F. Tabakin, Phys. Rev. C 73, 055204 (2006).

14. M. Guidal, J. M. Laget and M. Vanderhaeghen, Nucl. Phys. A 627, 645 (1997). 
15. T. Corthals, J. Ryckebusch and T. Van Cauteren, Phys. Rev. C 73, 045207 (2006), T. Mart and T. Wijaya, Acta Phys. Polon. B 34, 2651 (2003).

16. T. Mart and C. Bennhold, Phys. Rev. C 61, 012201 (1999).

17. F. X. Lee, T. Mart, C. Bennhold and L. E. Wright, Nucl. Phys. A 695, 237 (2001); B. S. Han, M. K. Cheoun, K. S. Kim and I. T. Cheon, Nucl. Phys. A 691, 713 (2001); A. de la Puente, O. V. Maxwell and B. A. Raue, arXiv:0809.3805 [nucl-th].

18. R.A. Adelseck and B. Saghai, Phys. Rev. C 42, 108 (1990).

19. M. Q. Tran et al., Phys. Lett. B 445, 20 (1998).

20. K. H. Glander et al., Eur. Phys. J. A 19, 251 (2004)

21. R. Bradford et al., Phys. Rev. C 73, 035202 (2006)

22. M. Sumihama et al., Phys. Rev. C 73, 035214 (2006)

23. K. Hicks et al., Phys. Rev. C 76, 042201 (2007).

24. A. Lleres et al., Eur. Phys. J. A 31, 79 (2007)

25. R. Lawall et al., Eur. Phys. J. A 24, 275 (2005)

26. H. Kohri et al., Phys. Rev. Lett. 97, 082003 (2006)

27. R. Bradford et al., Phys. Rev. C 75, 035205 (2007).

28. A. Lleres et al., Eur. Phys. J. A 39, 149 (2009)

29. R. M. Mohring et al., Phys. Rev. C 67, 055205 (2003)

30. D. S. Carman et al., Phys. Rev. Lett. 90, 131804 (2003)

31. P. Ambrozewicz et al., Phys. Rev. C 75, 045203 (2007)

32. T. Mart and A. Sulaksono, Phys. Rev. C 74, 055203 (2006)

33. P. Bydžovský and T. Mart, Phys. Rev. C 76, 065202 (2007).

34. T. Mart, Few Body Syst. 42, 125 (2008).

35. S.B. Gerasimov, Sov. J. Nucl. Phys. 2, 430 (1966); S.D. Drell and A.C. Hearn, Phys. Rev. Lett. 16, 908 (1966).

36. A. V. Anisovich et al., Eur. Phys. J. A 34, 243 (2007)

37. M. Bockhorst et al., Z. Phys. C 63, 37 (1994).

38. S. Capstick and W. Roberts, Phys. Rev. D 49, 4570 (1994); 58, 074011 (1998).

39. C. Caso et al., Eur. Phys. J. C 3, 1 (1998).

40. S. Janssen et al., Eur. Phys. J. A 11, 105 (2001)

41. A. Martinez Torres, K. P. Khemchandani, U. G. Meissner and E. Oset, arXiv:0902.3633 [nucl-th] (2009).

42. R. Nelson and T. Mart, arXiv:0904.3598 [nucl-th], to be published.

43. T. Mart and B. I. S. van der Ventel, Phys. Rev. C 78, 014004 (2008); T. Mart, Nucl. Phys. A 815, 18 (2009).

44. K. Ohta, Phys. Rev. C 40, 1335 (1989).

45. H. Haberzettl, Phys. Rev. C 56, 2041 (1997).

46. H. Haberzettl, C. Bennhold, T. Mart, and T. Feuster, Phys. Rev. C 58, R40 (1998).

47. T. Mart, C. Bennhold, H. Haberzettl, and L. Tiator, KAON-MAID, available at http://www.kph.uni-mainz.de/MAID/kaon/kaonmaid.html.

48. A. Salam et al., Phys. Rev. C 74, 044004 (2006).

49. K. Tsukada et al., Phys. Rev. C 78, 014001 (2008).

50. T. Mart, Mod. Phys. Lett. A 23, 3317 (2008)

51. T. Mart and A. Sulaksono, arXiv:nucl-th/0701007 (2007). 